\title{
A Synthesis Approach to Understanding Repeated Peptides Conserved in Mineralization Proteins
}

\author{
Kiyotaka Shiba ${ }^{*} \neq$ and Tamiko Minamisawa ${ }^{\dagger}$ \\ ${ }^{\dagger}$ Department of Protein Engineering, Cancer Institute, Japanese Foundation for Cancer Research, \\ Koto-ku, Tokyo 135-8550, Japan, ${ }^{\ddagger}$ CREST, JST
}

\section{Supporting Experimental Procedures}

Construction and Purification of artificial proteins. The artificial proteins used in this study were created using the MolCraft system. ${ }^{1}$ First, MG-44 and MG-55 were designed so that they encoded the nacrein motif NGNNGDNGN (or NNGNNGDNG) in their first reading frame and did not contain a termination codon within any of their three reading frames or at junctions. Based on the designer sequences, two pairs of MPR primers, KY-1293 5' -TGCAAGCCGAGCGGAAAGTCTTGCAAGGGCGTA TGA-3' \& KY-1295 5'-GTTCCCATTGTCCCCATTGTT CCCATTTCTCCATACGCCCTTA-3', and 5'-GTCTGG GTCGACGAATCCGGGAAAGTTGTTGTTTA-3' \& 5' -CCCGTTGTCCCCATTGTTCCCATTGTTCGAAACA ACAAA-3' were synthesized. Note that they have double mismatch pairs at their 3'-OH ends. These enhanced the polymerization reaction during MPR. ${ }^{2}$ Using these primer pairs, thermal cycle reactions were repeated in the presence of a thermostable archaeal DNA polymerase and dNTP (but without any template DNA) to polymerize the microgenes. Under these MPR conditions, nucleotide insertions and deletions randomly occur at end-joining junctions between microgenes, resulting in preparation of combinatorial libraries of three reading frames from a single microgene. ${ }^{2}$ The obtained libraries were cloned into the SmaI site of pTZ19R ${ }^{3}$ for sequence determination.

For protein expression, BamHI-Asp 718 fragments from polymer clones in pTZ19R were subcloned into the $B g l \mathrm{II}-$ Asp718 sites of expression vectors pKS600-605. Each of these vectors can mediate translation one of the six coding frames of the microgene polymers as an N-terminal Histagged fusion protein and were constructed as follows. First, the SalI-HindIII fragment of pKS583 ${ }^{4}$ was cloned into pQE-9, 10 or 11 (Qiagen), yielding pKS589, 590 or 591, respectively. The SalI-SmaI fragments of pKS589-591 were replaced with an adaptor, 5'-TCGACAGATCTGGAGCT CGGTACCC-3'+5' - AGATCTCGAGCTCGGTACCG-3', to produce pKS600, 602 or 604, respectively. Similarly, replacement with the adaptor 5'-TCGACGGTACCGAGC TCGAGATCT-3'+5' -AGATCTCGAGCTCGGTACCG-3' yielded pKS601, 603 or 605 , respectively. Each of the resultant expression vectors contained a T5 promoter, a translation initiation site, an oligo-histidine tag polypeptide, BglII-Asp 718 or Asp 718-BglII sites, a universal termination sequence, and a transcriptional terminator signal.
The subcloned polymers were expressed in E. coli $\mathrm{K} 12$ strain XL1Blue (Stratagene), and cell extracts were analyzed by SDS polyacrylamide gel (10-20\% gradient gel, Daiichi Pure Chemicals) followed by Coomassie brilliant blue R250 staining (Figure S7). Proteins were purified using TALON resin (Clontech) under denatured conditions, as described previously, ${ }^{4}$ and were dialyzed against $50 \mathrm{mM}$ Tris-acetate (pH 4.0), $100 \mathrm{mM} \mathrm{NaCl}$ and $1 \mathrm{mM}$ EDTA. The identities of the purified proteins were confirmed by determining their molecular weights using mass spectrometry (Ciphergen, data not shown).

\section{Results}

Periodic structures observed in biomineralization proteins. Aspein (Figure S5A) from pearl oyster contains repeats of a pentapeptide and its derivatives. ${ }^{5}$ SM50 (Figure S5B) from the sea urchin spicule contains mixtures of repeats of a hexapeptide and a heptapeptide,${ }^{6}$ while starmaker (Figure $\mathrm{S} 5 \mathrm{C}$ ) from the zebrafish otolith contains tandem three repeats of 13-amino acid sequences in its N-terminal domain. ${ }^{7}$ Most of the structures of mucoperlin (Figure S5D) from mussel shell and MAM22 (Figure S5E) from a magnetic bacterium are respectively filled with repeats of 31- and 34-amino acid sequences. ${ }^{8,9}$ MSP-1 (Figure S5F) from scallop shell and lustrin A (Figure S5G) from abalone nacre show convoluted repetitive structures. ${ }^{10,11}$

Repeats in Nacrein proteins. Human carbonic anhydrase II, one of the closest homologues to nacrein, by X-ray crystallography revealed that the structure contains a twisted $\beta$-sheet composed of ten $\beta$-strands (Figure S6A). From multiple alignments of related sequences, it can be discerned that the inserted domain is located somewhere between strands 7 and 8 , starting in the vicinity of the active site and encircling to the backside of the active site (Figure S6B). Nacrein form P. fucata is that it is composed of simple repeats of Asn-Gly-Asx (NGX). Intestingly, a nacrein from the Australian pearl oyster $P$. maxima contains an inserted domain that is much longer ( 245 amino acids) than the one from $P$. fucata. $^{12}$ The repeated sequences include $36 \mathrm{x}$ $\mathrm{NGN}, 4 \mathrm{x}$ NGD, $4 \mathrm{x}$ NGY and $4 \mathrm{x}$ NGG, as well as repeats of a dipeptide (6 $\mathrm{x}$ GN) that is not found in P. fucata. This repeated GN dipeptide dominates within the $\sim 235$-amino acid inserted domain of the nacrein isolated from Turbo marmoratus (turban shell). ${ }^{13}$

Artificial proteins containing mineralization motifs. Some clones were well expressed in E. coli (Figure S7A and B). Six proteins were purified (Figure S7C). 


\section{A Aspein}

MKGIAILMCLAALVAVSVTFPVADQTTNELGSSGA AGAVVSEPSDAGDAADAGDADAADADAADADADA ADADIIDDGDDDDDDDDDDSGDDDSGDDDDSGDDDD GDDDDSGDDDDSGDDDDSGDDDGDDDSEDDDDSGD DSGDGDDGDSGDDDDDDDSGDDDDDDDSGDDDDDS DDDEDSGDDDSGDDDGDDDDSGDDDSGDDDDSGD

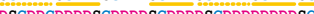
DDDGDSEDDSGDDDGDDDDSGDDSGDESGDDDS DDDSGDDDSGDDDSGDDDSDSGDDDSGDDDSGD GDDDGDDGDDDADSGDDDDDDDDDDGDDGDDDSG DDGDDSDDDDDDDDDDQ

\section{B $5 M 50$}

MKGVLFIVASLIAFATGQDCPAYYVRSQSGQSCYRY FNMRVPYRMASEFCEMVTPCGNGPAKMGALASVSSP QENME IYQLVAGFSQDNQMENEVWLGWNSQSPFFW DGTPAYPNGFAAFSSSPASPPRPGMPPTRSWPVNPQ NPMSGPPGRAPVMKRQNPPVRPGQGGRQI PQGVGPQ MPC $M$ M NQQPGMGGRQPGFGNQPGMGGRQPGFGNQPGMGGR GGQPGVGGROPGFGNOPGMVDNNOAWWTTTRLGNO VGGRQPGMGGQPGVGGRQPGVGGRQPGFGNQPGV GRQPGMGQQPGMGGQPGVGGRQPGMGGRQPGFGNQ

\section{Starmaker}

APVSNNNGTDNDESAADQRHIFTVQFNVGTPAPAD DSVTTDGKDSAEKNEAPGDSSDTTEKPGTTDGKDS EQHGVTTDGKDEAEQHGVTTDGQDSAEKRGEADGA DKPDTQNGTDDTDSDKETDASHHKTGDSDENKDKP DG SSASAEKSD DKSOEKSDKSDDGSSEADERESVESKDHDSDSQ SDSAEKKEKHDDKDQDSSDSADSKDSDEDKDKDHSE QKDSEDHEHKEKHTKDKEEHKDSDEGKDDEDKSKS EHDKDESESKEASKSDESEQEEKKDDKSDSDNSSR SHSDSDSDSHSDSDSDSHSDSHSDSDSDSHSDSDSD SDSDSDSDSDSDSDSNSRDKEEKKDKSSESRDEDSS DSTS SERE DVSDDDDIDAHDDEGVEHGTDEASKPHOEPDH DTTHGSDDGSKTSMPIS

\section{Mucoperlin}

MAAVSLTGTLALSSGMPLSKPMPDSTQVVEIPIQS SLLQPKLSSSGMPLSKPMTGSTQVVEIPIQSAGLLQ KLSSGMPLSKPMPDSTQVQIPIQSAGIMQPKLSS PMP PQVDTRIQSEIMOPKISEMPISKPMDSPEVI IPIOSAGLLOPILSSGMPISKPITDSPOVVETPIOS AGILPPNLSSEMPLSKPITDSPQVVEIPIQSAGIL PNLSSEMPLSKPITDSPQVVETPIQSAGILPPNLS EMPLSKPI TDSPQVVEIPIQSAGILPPNLSSEMPI PITDSPQVEIPIQSASILQPKLSSEMPLSKPMP QQVVGKHQSAGKMQSSVLTTSGPAAVPVAAGSLS

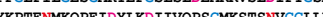
DDIEVKTSGOEDYIKSSDVTRPGNIGIMSMLGPSM EDGMVNLAEGELSALKKRDSTMGTVYTTLAGDV RDKDATYPVTRNDAVFKPECINGLGPPCKLAVTSR AAVPVVSGVSLTNDTKVPCDKWNN

\section{E MAM22}

MSSKPSDILDEVTLYAHYGLSVAKKLGMNMVDAFRA AFSVIDDIRQVYYRDKGISHAKAGRYSQAVMLLEQV DAPDNVKVATVLCITYVOVOKYDIAVPUITVMEA PINFNVRFRLGVALDNLGRFDEAIDSFKIALGLRP $\mathrm{V}$ GKVHRAIAFSYEQMGRHEEALPHFKKANELDEGAS

\section{F MSP-1}

MYIKLILGVLALVALAVSAPLDTDKDLEFHLDSLL AAEDGGGGDAAGAEKAAPAADLSGGSKGGSKGSST SSKGGSKGGSKGGNGGEDADDSSSSSGSDSGSSGS EESDDSSSSSSSGSGGSGSSGSSGSGSSSSSGSSSDG SDDGSDDGSDSGDDADSANADDLDSNDSDDSDNSGS SDDSDDSDDSSNDVNESNSDESGPGGYGGNGPAG GKGRKGGVGCNGGNGGDGSSSSSSGSCSGSTS SSSGSGSGSGSGSGSSSSSSSSGDGSDDGSDDGS SGDDANSANADDLDSNDPDDSDNSGSNGESDSDNSS SDDGDGSDSGSDSGRDSQSDDASNNDSDDSDDSDN STDTGESDSDESGPGGYGGNGPAGNGGKGRKGGNGG GNGGGNGGNGGDGSSSSASSGSGSGSGSGSGSGSG ANSANADDLDSNDPDDSDNSGSNGESDSDNSSSDDG DGSDSGSDSGRDSOSDASINDSDSSDSDNSSTD GESDSDESGPGGYGGNGPAGNGGKGRKGGKRGGNG NGNGGNGGDGSSSSSSSGSGSGSDSGSGSSSGSGS SGSSSSSSSSGDGSDDGSDDGSDDGDDANSANADD DSNDPDDSDNSGSNGESDSDNSSSDDGDGSDSGSD GRDSOSDDASNNDSDDSDDSDNSSTDTGESDSDES PGGYGGNGPAGNGGKGRKGGKGGRGGNGGNGGGGS

\section{G Lustrin A}

MERFLWVLCIAAGFSVNYGLRRAPYPCEPGLNVNCT TIDTNLEISCAPDGSCPATTGCVVPS INCTIGK FNPSSGPPGPPRPPGPPRPPGPPQPDPNLLDPCFP KNVNCTSGECRLMADCQHOSCPALPYCVAPSPNVTV PCPIGKSAIDRNLREFSCLRNRDACPRSTGCVVGA

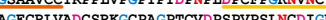
PALNSYGNEISCAGG ACPVNTVCVAHPSCAPAVCC FKPAGPTTPQPPTIPQPPTTPSSPTGDPCEPGNVI CTAGTCRLVVDCRFPGCPAVPKCVDPSSKPSLNCS GDPALNPNLQEISCVGGAACPRNTACFAAPSGSPAV CCYTSGPPRPEPPSPSPPTGDPCEPGVNVNCTAGTC RLVEDCRIRGCPAVPKCIDRDPPLPPPPDVCPVGT VLGADLKQLYCGRGGRRCPWNTYCVIHPADRYAVCC VANCDYWPCPARPTCYDHSPEPSIN VANCDYWPCPARPTCVDHSPEPSLNCTIGDPALNGX ITPAPTTVPIPVSTAAPTSAPGPVDPCQPGVNVNCT KGECRLVAICKYWPCFALPTCVDPSPPPSVECPVG PALDEKLEEFSCKDCPENTVCYKGAVCCVPWSGNR SGPAGPAGPAGPERPATSVPLDPCTPGLNVNCTSG CRLVEDCRRPGCPAVPICIDRDPPLPPPPDVCPVG PVLGRDLKQLYYCGRGGKRCPGNTYCVIHPADRYAVC LVAYCNAVPCGRTTPTCVDPSPPPTRKCPVCKPVG PRLTEFRCYPRVRICPGDSFCIRGPGDEPGCCWD RLRPTQGGGSGSGSGSGSGSSSSSSSGSTSGSGSG GSGSSSGSGSGSSSASGSGSSSGSSSGSSSGSSSGS SSGSGSGSGSGSSSASGSGSSSGSSSGSSSGSGSG SSASGSGSSSGSGSGSSSGSGSGSGSGSSSGSGSGS SSGSSSVSNSWTGSGSSSGSGSGSSSWSGSGSSSG

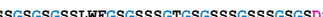
SSGSSSGSTSGSSSGSGSASGSGTGSGKGASYDTD DSGSDNRSPGYLPODPCTPGLYINCTAGTCRLTAWC SNFCPAVPTCVDSSPDASGECPVGLPALNYFN SCRTNLQCPSNTYCKSPGICCYRGPIARPRSSRYLA KYLKQGRSGKRLQKPGSCPAVRPDWAGICVVRCFCD
A

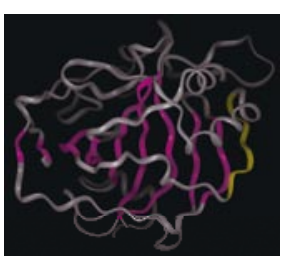

B

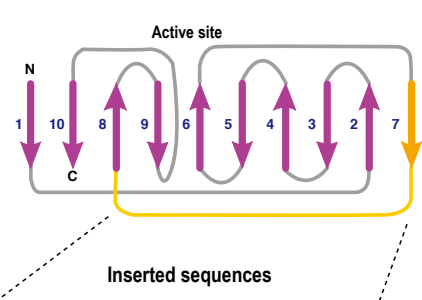

Pinctada fucata

HHPDNNENGNGDNGNNGYNRGDNGNNGDNGNNSYN DNGNNGVNGNNGYNGDNGNNGDNGNNGYNGDNGNN DNGNNGENGNNGENGNNGENGH

HCENNGDNGNNCDNGNNGNNDNNGNNGNNGNGNNGY NGNNGDNGANCW NDNNGNDNNGNNGGNGNNGNNGNGNNGNNGNGNNG NGGNGNNGNNGNSNNGNNGNGNNGNNGGNGNNGNNG NGNNENNGNGSNGNNGGNGNNGNNGDNGNGDNGYN DNGNSDGRLRRWDLANVRRMHAERYHFSGGCIVKKA KRLSRILECAYRHKKVREFKRNGEEKGL

RKFFRSCRRRIYSADYFKKYYGD GDNGNGNGNGNGN GNGKDNGNGNGNDNGY GNGNGNGNGNGNGNGNGNG GNGYGYGNGNGNGNGNGNGNGNGNGNGNGNGNGNGY

Turbo marmoratus 作 GNGNGNGNGNGNGKDNGDNSNYGDEEDDDIYGFCRR YCDDDQCPDDAEPECLDGD

Figure S6. Appendix domains composed of simple repeats in nacrein. (A) Structure of human carbonic anhydrase II.14 $\beta$-strands that form a twisted $\beta$-sheet in the enzyme that are shown in magenta, except strand 7 is shown in yellow. (B, top) Schema representing the topology of the $\beta$-strands forming the central twisted sheet. Multiple sequence alignments between central twisted sheet. Mutiple sequence alignments between mammalian carbonic anhydrase and nacreins suggest that an additional domain is inserted somewhere between strands and 8 and is shown in yellow. (B, bottom) Sequences of the maxima and Turbo marmoratus are shown. Asparagine, asparate and glycine are shown in red, magenta and cyan, respectively.

A

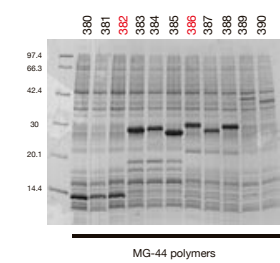

B
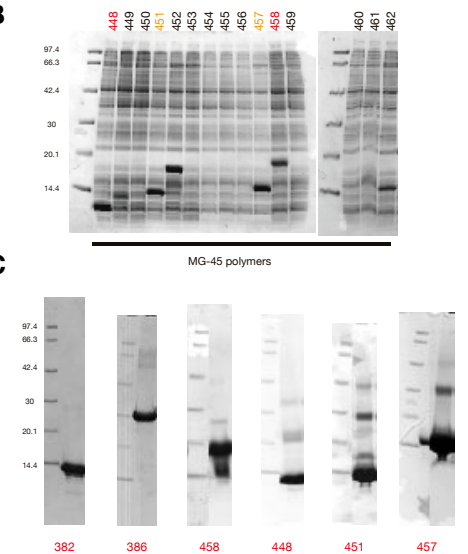

Figure S7. Artificial proteins created in this study. (A) Cell extracts from $E$. coli XL1Blue harboring clones of MG-44 polymers were analyzed by $15 / 25 \%$ gradient SDS-PAGE. The numbers of the pMT clones are shown at the top; the molecular weights $\left(x 10^{-3}\right)$ of standard makers are shown on the left. (B) Similar to (A) but for MG-45 polymers. (C) Purified artificial proteins were run in $15 / 25 \%$ gradient SDS-PAGE and stained with Coomassie brilliant blue. Molecular weights $\left(x_{10}^{-3}\right)$ of standard makers are shown on the left. Although some images were trimmed by digital manipulations, standard makers were always run within the same gel. 


\section{K. Shiba \& T. Minamisawa}

Inhibitory effects of artificial proteins on in vitro calcification. A synthetic 9-mer peptide (NGN-NGDNGN) caused no significant delay in the drop in $\mathrm{pH}$, indicating that the isolated peptide motif was not sufficient to suppress $\mathrm{CaCO}_{3}$ formation in this assay (Figure S8). At the ambient temperature, the suppressive effect of the \#382 was concentration-dependent (Figure S9A). This suppressive effects exerted by $20 \mu \mathrm{g}$ of \#382 were diminished as the assay temperature was increased from $25^{\circ} \mathrm{C}$ to $40^{\circ} \mathrm{C}$ (Figure S9B). At $40^{\circ} \mathrm{C}$, both the time to onset and the rate of decline in $\mathrm{pH}$ were indistinguishable from those observed in a control experiment with no inhibitory protein (Figure S9C).

Other unstructured (\#451) and structured (\$57) artificial proteins created from MG-45 did not suppress calcification (Figure S10). Also, three unstructured artificial proteins created from microgenes other than MG-44 and MG-45 failed to exhibit suppressor activity (Figure S11).

FTIR analyses of calcification products. Fourier transform infrared spectroscopy spectra suggested that the calcification products were calcite (Figure S12).

Effect of buffer constituents on calcification assay. Because buffer constituents interfered with the calcification assay (Figure S13), we extensively dialyzed proteins before their use in the assays.

A

NR-AsnGIYAsnAsnGIYAspAsnGlyAsn-coor

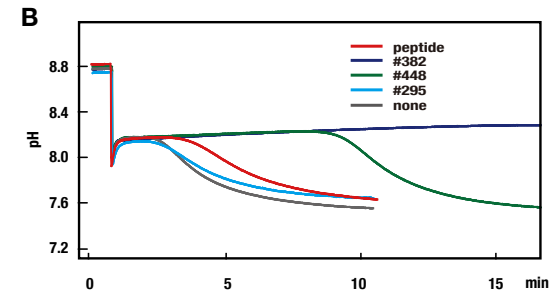

Figure S8. Inhibition of $\mathrm{CaCO}_{3}$ formation by artificial proteins. (A) Sequence of the peptide. (B) Capacity of the artificial proteins \#295 (see Figure S 10), \#382 and \#448 to inhibit $\mathrm{CaCO}_{3}$ proteins \#295 (see Figure $S 10$ ), $\# 382$ and $\# 448$ to inhibit $\mathrm{CaCO}_{3}$
formation. The assay was carried out at $20^{\circ} \mathrm{C}$ with $20-\mu \mathrm{g}$ samples of protein.
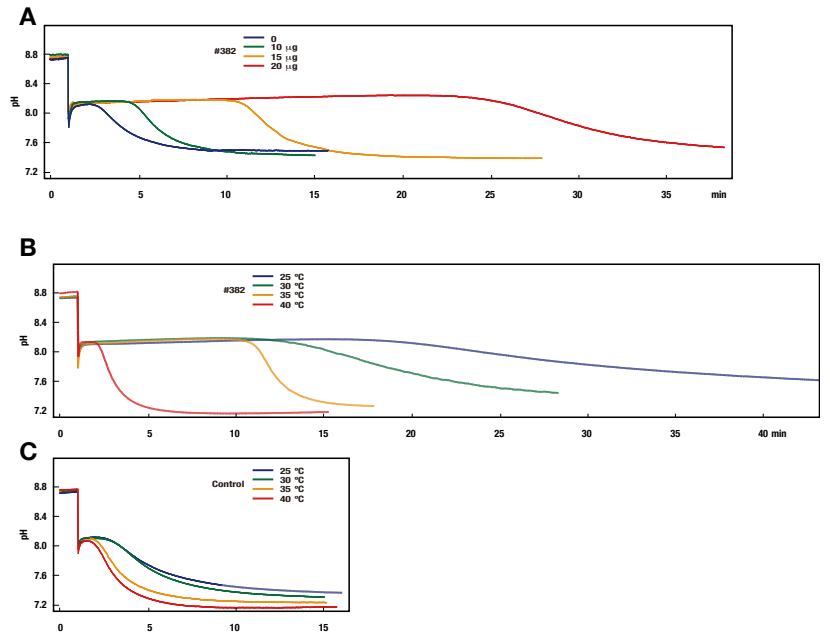

Figure S9. Effects of the assay conditions on evoked declines in $\mathrm{pH}$. (A) Effects of the amount of protein were assessed by adding 10,15 or $20 \mu \mathrm{g}$ of \#382 to the assay mixture. Ambient temperature was used in this assay. Effects of temperature were assessed using $20 \mu \mathrm{g}$ of \#382 (B) and control $\left(\mathrm{H}_{2} \mathrm{O}, \mathrm{C}\right)$.
Biomacromolecules

A

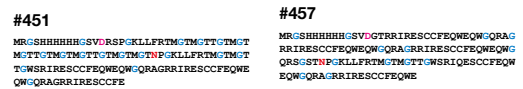

B

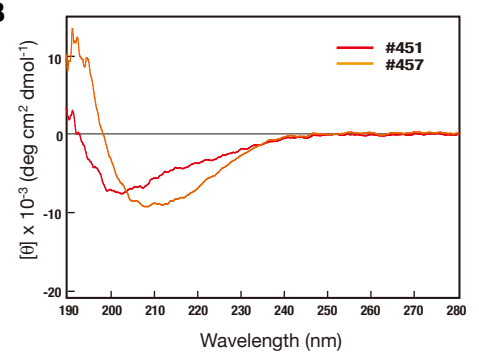

C

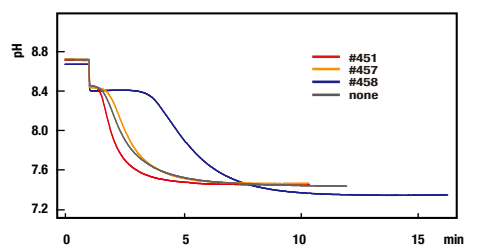

Figure S10. Properties of other artificial proteins created from MG-45. (A) The amino acid sequences of two proteins obtained from a MG-45 polymer library. Asparagine, Asparate and glycine are shown in red, magenta and cyan, respectively. (B) Far UV CD spectra of \#451 and \#457. Conditions are same as described in the legend to Fig. 3. (C) Capacities of \#451 and \#457 to inhibit $\mathrm{CaCO}_{3}$ formation. For comparison, \#458 (inhibitor, Figure $\mathrm{S} 5$ ) was included. The assays were carried out at $25^{\circ} \mathrm{C}$ with $10-\mu \mathrm{g}$ samples of protein.

A

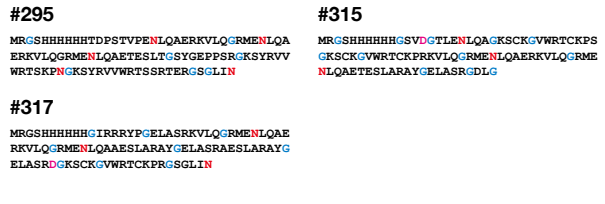

B
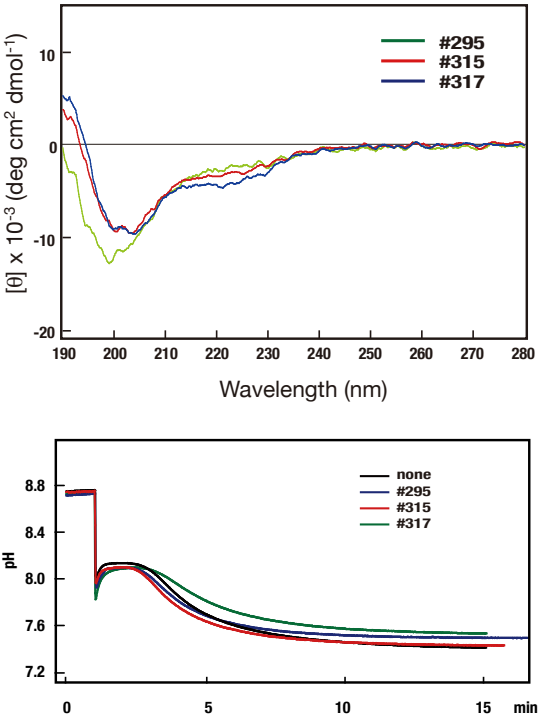

Figure S11. Properties of artificial proteins created from microgenes other than MG-44 and MG-45. (A) Amino acid sequences of three proteins obtained from a MG-33 (for \#315 and \#317) and MG-35 (for \#295) polymer libraries. Asparagine, aspartate and glycine are shown in red, magenta and cyan, respectively. (B) Far UV CD spectra of \#295, \#315 and \#317. Conditions are the same as described in the legend to Fig. 3. (C) Capacities of \#295, \#315 and \#317 to inhibit $\mathrm{CaCO}_{3}$ formation. Assays were carried out at $25^{\circ} \mathrm{C}$ using $3.3-\mu \mathrm{g}$ samples of protein. 


\section{Control}

\#382

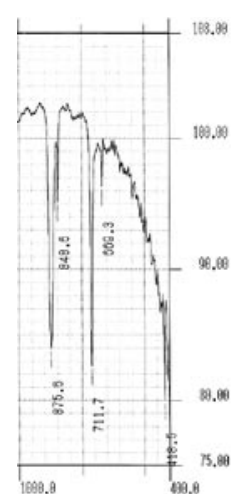

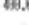
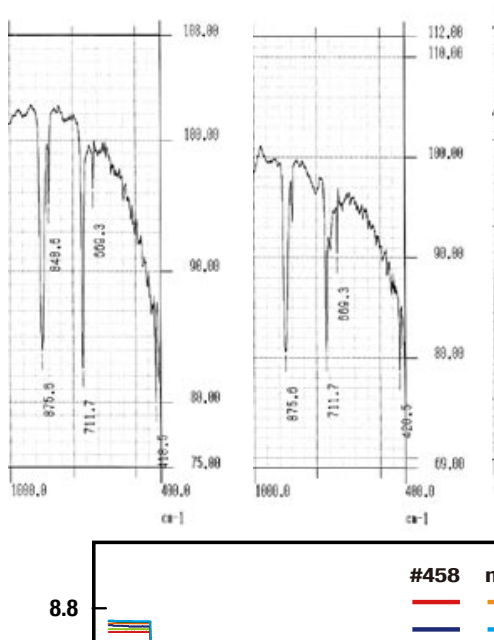

\#386

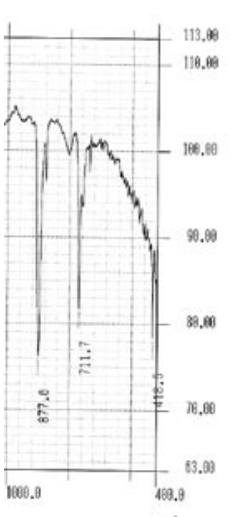

$\$ 458$ none

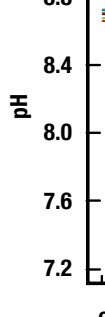

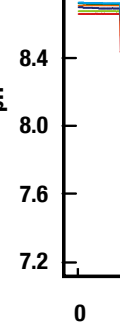

\#458

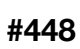

\#448

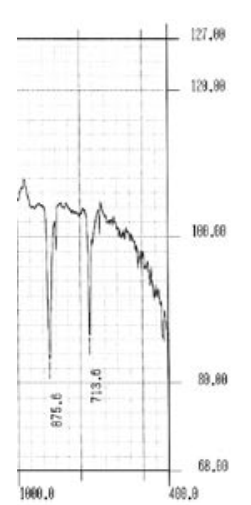

a-1

Figure S12. Fourier transform infrared spectroscopy (FTIR) spectra of $\mathrm{CaCO}_{3}$ precipitants obtained after incubating precipitants obtained after incubating $20-\mu \mathrm{g}$ samples of artificial protein for a week. The abscissa and longitudinal axes represent frequency $\left(\mathrm{cm}^{-1}\right)$ and transmission (\%), respectively. The spectra were obtained using a Shimazu FTIR-8200A spectrophotometer. The peak at around $876 \mathrm{~cm}^{-1}$ is specific to calcite (aragonite should give a peak at around $\left.857-860 \mathrm{~cm}^{-1}\right) .15,16$

Figure S13. Effects of the buffers used for protein dialysis. Samples 110 $\mu \mathrm{g})$ of \#458 were assayed at $25^{\circ} \mathrm{C}$ in water, in $3 \mathrm{mM} H \mathrm{HEES}-\mathrm{NaOH}(\mathrm{pH}$ 8.7) and in $3 \mathrm{mM}$ TrisHCl (pH 8.7).

\section{References}

(1) Shiba, K., MolCraft: a hierarchical approach to the synthesis of artificial proteins. J. Mol. Catlys. B 2004, 28, 145-153.

(2) Shiba, K.; Takahashi, T.; Noda, T., Creation of libraries with long open reading frames by polymerization of a microgene. Proc. Natl. Acad. Sci. U S A 1997, 94, 3805-3810.

(3) Mead, D. A.; Szczesna-Skorupa, E.; Kemper, B., Single-stranded DNA 'blue' T7 promoter plasmids: a versatile tandem promoter system for cloning and protein engineering. Prot. Eng. 1986, 1, 67-74.

(4) Shiba, K.; Takahashi, Y.; Noda, T., On the role of periodism in the origin of proteins. J. Mol. Biol. 2002, 320, 833-840.

(5) Tsukamoto, D.; Sarashina, I.; Endo, K., Structure and expression of an unusually acidic matrix protein of pearl oyster shells. Biochem. Biophys. Res. Commun. 2004, 320, 1175-1180.

(6) Katoh-Fukui, Y.; Noce, T.; Ueda, T.; Fujiwara, Y.; Hashimoto, N.; Higashinakagawa, T.; Killian, C. E.; Livingston, B. T.; Wilt, F. H.; Benson, S. C.; et al., The corrected structure of the SM50 spicule matrix protein of Strongylocentrotus purpuratus. Dev. Biol. 1991, 145, 201-202.

(7) Sollner, C.; Burghammer, M.; Busch-Nentwich, E.; Berger, J.; Schwarz, H.; Riekel, C.; Nicolson, T., Control of crystal size and lattice formation by starmaker in otolith biomineralization. Science 2003, 302, 282-286.

(8) Okuda, Y.; Denda, K.; Fukumori, Y., Cloning and sequencing of a gene encoding a new member of the tetratricopeptide protein family from magnetosomes of Magnetospirillum magnetotacticum. Gene 1996, 171, 99-102.
(9) Marin, F.; Corstjens, P.; de Gaulejac, B.; de Vrind-De Jong, E.; Westbroek, P., Mucins and molluscan calcification. Molecular characterization of mucoperlin, a novel mucin-like protein from the nacreous shell layer of the fan mussel Pinna nobilis (Bivalvia, pteriomorphia). J. Biol. Chem. 2000, 275, 20667-20675.

(10) Sarashina, I.; Endo, K., The complete primary structure of molluscan shell protein 1 (MSP-1), an acidic glycoprotein in the shell matrix of the scallop Patinopecten yessoensis. Mar. Biotechnol. (NY) 2001, 3, 362-369.

(11) Shen, X.; Belcher, A. M.; Hansma, P. K.; Stucky, G. D.; Morse, D. E., Molecular cloning and characterization of lustrin A, a matrix protein from shell and pearl nacre of Haliotis rufescens. J. Biol. Chem. 1997, 272, 32472-32481.

(12) Kono, M.; Hayashi, N.; Samata, T., Molecular mechanism of the nacreous layer formation in Pinctada maxima. Biochem Biophys Res. Commun. 2000, 269, 213-218.

(13) Miyamoto, H.; Yano, M.; Miyashita, T., Similarities in the structure of nacrein, the shell-matrix protein, in a bivalve and a gastropod. $J$. Mollus. Stud. 2003, 69, 87-89.

(14) Eriksson, A. E.; Jones, T. A.; Liljas, A., Refined structure of human carbonic anhydrase II at 2.0 A resolution. Proteins 1988, 4, 274-282.

(15) Falini, G.; Albeck, S.; Weiner, S.; Addadi, L., Control of aragonite or calcite polymorphism by mollusk shell macromolecules. Science 1996, 271, 67-69.

(16) Choi, C. S.; Kim, Y. W., A study of the correlation between organic matrices and nanocomposite materials in oyster shell formation. Biomaterials 2000, 21, 213-222. 